Article

\title{
The Porcine Nasal Microbiota with Particular Attention to Livestock-Associated Methicillin-Resistant Staphylococcus aureus in Germany-A Culturomic Approach
}

\author{
Andreas Schlattmann ${ }^{1}$, Knut von Lützau ${ }^{1}$, Ursula Kaspar 1,2 and Karsten Becker 1,3,*(D) \\ 1 Institute of Medical Microbiology, University Hospital Münster, 48149 Münster, Germany; \\ andreas.schlattmann@ukmuenster.de (A.S.); knutlvl@gmail.com (K.v.L.); Ursula.Kaspar@lzg.nrw.de (U.K.) \\ 2 Landeszentrum Gesundheit Nordrhein-Westfalen, Fachgruppe Infektiologie und Hygiene, \\ 44801 Bochum, Germany \\ 3 Friedrich Loeffler-Institute of Medical Microbiology, University Medicine Greifswald, \\ 17475 Greifswald, Germany \\ * Correspondence: karsten.becker@med.uni-greifswald.de; Tel.: +49-3834-86-5560
}

Received: 17 March 2020; Accepted: 2 April 2020; Published: 4 April 2020

check for updates

\begin{abstract}
Livestock-associated methicillin-resistant Staphylococcus aureus (LA-MRSA) remains a serious public health threat. Porcine nasal cavities are predominant habitats of LA-MRSA. Hence, components of their microbiota might be of interest as putative antagonistically acting competitors. Here, an extensive culturomics approach has been applied including 27 healthy pigs from seven different farms; five were treated with antibiotics prior to sampling. Overall, 314 different species with standing in nomenclature and 51 isolates representing novel bacterial taxa were detected. Staphylococcus aureus was isolated from pigs on all seven farms sampled, comprising ten different spa types with $\mathrm{t} 899(n=15,29.4 \%)$ and $\mathrm{t} 337(n=10,19.6 \%)$ being most frequently isolated. Twenty-six MRSA (mostly t899) were detected on five out of the seven farms. Positive correlations between MRSA colonization and age and colonization with Streptococcus hyovaginalis, and a negative correlation between colonization with MRSA and Citrobacter spp. were found $(p<0.05)$. Of 209 non-S. aureus members of the Staphylococcaceae family, 25 isolates $(12.0 \%)$ from three out of the seven farms exhibited methicillin resistance, including two Macrococcus goetzii isolates carrying the mecB gene. Among 125 Enterobacterales, none tested positive for extended-spectrum beta-lactamase (ESBL) and carbapenemase production. The high frequency of methicillin-resistant staphylococci supports the need for enhanced efforts within the "One Health" concept to manage the antibiotic resistance crisis in the human and veterinary medicine sector.
\end{abstract}

Keywords: Staphylococcus; MRSA; microbiota; pig; Macrococcus; ESBL; Enterobacterales; Firmicutes; Proteobacteria; Actinobacteria

\section{Introduction}

Resistance to antimicrobial agents in microorganisms is on the rise and poses a global health threat. The World Health Organization (WHO) estimates that deaths due to antimicrobial resistance may increase from 700,000 to 10 million by 2050 if no strategies are implemented [1]. An important pathogen in this context is methicillin-resistant Staphylococcus aureus (MRSA): Almost 20,000 deaths were associated with 120,000 bloodstream infections in the USA alone in 2017 [2]. While these numbers are generally lower in Germany, a mortality rate of $6.4 \%$ has still been reported for the same year by the Robert Koch Institute [3]. 
In order to combat colonization and eliminate reservoirs of antibiotic-resistant bacteria, novel approaches are called for. One of these is the investigation of the microbiota that could lead to discoveries with regard to synergistic or antagonistic interactions within microbial communities of certain habitats $[4,5]$.

Habitats of particular interest are the nasal cavities of pigs because they can harbor livestock-associated MRSA (LA-MRSA). This subgroup of MRSA can colonize both humans and pigs, and occurrence of the predominant clonal complex (CC) 398 is particularly high in regions with intensive pig farming [6-8]. Moreover, LA-MRSA also colonizes healthy companion animals and horses without contact to livestock, which indicates that a spread to other animals has commenced [9-11]. Of note, zoonotic exchange of LA-MRSA between humans and animals has significant impact on the epidemiology of MRSA and contributes to the overall burden of MRSA colonization and infection in Germany and other countries [12-17].

Previous works have focused on molecular approaches, including metagenomic studies to elucidate the composition of the porcine nasal microflora. In addition to lower sensitivity, this approach does not allow for further functional metabolic characterization of hitherto undescribed bacterial taxa. We employed extensive culturomics in order to obtain data of viable, known and unknown bacterial species living in and on pig snouts. Although this approach has the caveat of not detecting microorganisms that do not survive the transport or are not able to grow on the culture media included in our design, the establishment of a biobank containing strains of the cultivable part of the porcine nasal cavity's microbiota offers the opportunity to validly describe novel taxa and to comprehensively characterize the generated isolates and make them available to the scientific community for future research.

\section{Materials and Methods}

Samples were processed and bacterial isolates identified as described previously [18]. In brief, swab samples of healthy pigs were collected and cultured on various media. Identification was performed using matrix-assisted laser desorption/ionization time-of-flight mass spectrometry (MALDI-TOF MS) and 16S rRNA gene sequencing. Resistance profiles for staphylococci and Enterobacterales were determined using VITEK ${ }^{\circledR} 2$ (bioMérieux, Marcy l'Etoile, France), and PCR was performed for resistance gene detection.

Fifty-four swab samples of 27 pigs from seven farms in the Münsterland region (northwestern Germany) using the transport swabs Transwab ${ }^{\circledR}$ in Amies medium (Medical Wire \& Equipment, Corsham, UK) were taken and delivered within $6 \mathrm{~h}$ to the Institute of Medical Microbiology of the University Hospital Münster. The sampled farms were located within German post code areas of 48xxx and participating farmers filled in a data sheet for their farms regarding total number of pigs, presence of other animals, farm type, and feed type (Table 1 ).

Swabs were vortexed in 0.9\% saline solution and 1:10, 1:100, and 1:1000 dilutions were prepared. Columbia agar with 5\% sheep blood, chocolate agar (both Becton Dickinson, Franklin Lakes, NJ, USA), Columbia CAP agar, and MacConkey agar (both Oxoid, Wesel, Germany) were inoculated with $100 \mu \mathrm{L}$ each of each dilution and incubated aerobically for $24 \mathrm{~h}$ (chocolate agar: 5\% $\mathrm{CO}_{2}$ ). Anaerobic incubation of Schaedler agar, Schaedler plus kanamycin/vancomycin agar (both Becton Dickinson, Franklin Lakes, NJ, USA), chocolate agar and Columbia CAP agar occurred after inoculation with the same amount of each sample for $48 \mathrm{~h}$. Single colonies were picked after the respective incubation time had elapsed.

MALDI-TOFMS was applied using the Microflex LT system, Biotyper 2.0 software, and flexControl version 3.4 (Bruker Daltonik, Bremen, Germany) for primary isolate identification, as previously described [19]. Briefly, single colonies were harvested and smeared on a ground steel target plate (Bruker Daltonik) in two replicates. Samples were covered with matrix solution. After co-crystallization, they were processed in the MALDI-TOF MS using FLEXCONTROL software (Bruker Daltonik) according 
to the manufacturer's instructions. Spectra were analyzed by MALDI BIOTYPER 4.1 (Bruker Daltonik) in the $\mathrm{m} / \mathrm{z}$ range of 4000-10,000 Da. Identification to the species level was assumed with scores $\geq 2.0$.

Table 1. Data of sampled farms.

\begin{tabular}{|c|c|c|c|c|c|c|}
\hline Farm & $\begin{array}{l}\text { Total Number } \\
\text { of Pigs }\end{array}$ & $\begin{array}{c}\text { Additional } \\
\text { Animals on Farm }\end{array}$ & $\begin{array}{c}\text { Feed } \\
\text { Consistency }\end{array}$ & Individual & $\begin{array}{l}\text { Age in } \\
\text { Months }\end{array}$ & Antibiotics \\
\hline \multirow{6}{*}{$\# 1$} & \multirow{6}{*}{1400} & \multirow{6}{*}{ Cattle } & \multirow{6}{*}{ Solid } & $\# 1$ & 0.5 & None \\
\hline & & & & $\# 2$ & 0.5 & None \\
\hline & & & & $\# 3$ & 0.5 & None \\
\hline & & & & $\# 4$ & 65 & None \\
\hline & & & & $\# 5$ & 39 & None \\
\hline & & & & $\# 6$ & 52 & None \\
\hline \multirow{3}{*}{$\# 2$} & \multirow{3}{*}{2000} & \multirow{3}{*}{ Dogs, cats } & \multirow{3}{*}{$\begin{array}{l}\text { Liquid and } \\
\text { pellets }\end{array}$} & $\# 1$ & 26 & None \\
\hline & & & & $\# 2$ & 19.5 & None \\
\hline & & & & $\# 3$ & 26 & None \\
\hline \multirow{3}{*}{$\# 3$} & \multirow{3}{*}{3800} & \multirow{3}{*}{ Dogs } & \multirow{3}{*}{ Liquid } & $\# 1$ & 52 & None \\
\hline & & & & $\# 2$ & 39 & Enrofloxacin \\
\hline & & & & $\# 3$ & 51.5 & Enrofloxacin \\
\hline \multirow{3}{*}{$\# 4$} & \multirow{3}{*}{1000} & \multirow{3}{*}{ Dogs, cats, horses } & \multirow{3}{*}{ Liquid } & $\# 1$ & 3 & None \\
\hline & & & & $\# 2$ & 3 & None \\
\hline & & & & $\# 3$ & 3 & None \\
\hline \multirow{3}{*}{$\# 5$} & \multirow{3}{*}{2200} & \multirow{3}{*}{ Dogs, horses } & \multirow{3}{*}{$\begin{array}{l}\text { Liquid and } \\
\text { solid }\end{array}$} & $\# 1$ & 2 & Amoxicillin \\
\hline & & & & $\# 2$ & 2 & Amoxicillin \\
\hline & & & & $\# 3$ & 2 & Amoxicillin \\
\hline \multirow{6}{*}{$\# 6$} & \multirow{6}{*}{1600} & \multirow{6}{*}{ Dogs } & \multirow{6}{*}{ Liquid } & $\# 1$ & 4 & None \\
\hline & & & & $\# 2$ & 4 & None \\
\hline & & & & $\# 3$ & 4 & None \\
\hline & & & & $\# 4$ & 35.5 & None \\
\hline & & & & $\# 5$ & 9.5 & None \\
\hline & & & & $\# 6$ & 29.5 & None \\
\hline \multirow{3}{*}{$\# 7$} & \multirow{3}{*}{1600} & \multirow{3}{*}{ Dogs, sheep } & \multirow{3}{*}{ Liquid } & $\# 1$ & 6 & None \\
\hline & & & & $\# 2$ & 6 & None \\
\hline & & & & \#3 & 6 & None \\
\hline
\end{tabular}

Extraction of DNA from bacteria with lower scores was performed with QIAamp DNA Mini Kit (Qiagen, Hilden, Germany) according to the manufacturer's instructions. REDTaq ${ }^{\circledR} \operatorname{ReadyMix}^{\mathrm{TM}}$ (Sigma-Aldrich Chemie GmbH, Munich, Germany) and primers SSU-bact-27f (5'-AGA GTT TGA TCM TGG CTC AG-3') and 16S-5 (5'-AAG GAG GTG ATC CAG CCG CA-3') were used for 16S rRNA PCR. Gene amplification was executed at $94{ }^{\circ} \mathrm{C}$ for $5 \mathrm{~min}, 28$ cycles of $45 \mathrm{~s}$ at $94{ }^{\circ} \mathrm{C}, 60 \mathrm{~s}$ at $60{ }^{\circ} \mathrm{C}$ and $90 \mathrm{~s}$ at $72{ }^{\circ} \mathrm{C}$, and finished with the final elongation at $72{ }^{\circ} \mathrm{C}$ for $10 \mathrm{~min}$. If amplification failed, primers SSU-bact-27f and 16S-2 (5'-CCG TCA ATT CMT TTG AGT TT-3') were used with amplification conditions of $95^{\circ} \mathrm{C}$ for $5 \mathrm{~min}, 30$ cycles of $60 \mathrm{~s}$ at $95^{\circ} \mathrm{C}, 60 \mathrm{~s}$ at $60{ }^{\circ} \mathrm{C}$ and 2 min at $72{ }^{\circ} \mathrm{C}$, and finished with the final elongation at $72{ }^{\circ} \mathrm{C}$ for $10 \mathrm{~min}$. The QIAquick PCR purification kit (Qiagen, Hilden, Germany) was used according to the manufacturer's instructions to prepare the amplicons for gene sequencing with primers SSU-bact-27f and 16S-5 or SSU-bact-27f and 16S-2. 
Cycle sequencing technology and subsequent analysis on an ABI 3730XL (Eurofins Genomics GmbH, Ebersberg, Germany) were employed to generate chromatograms which were assembled with DNA Sequence Assembler (Heracle BioSoft S.R.L., Pitești, Romania). Sequences were deposited in GenBank under accession numbers MT007966-MT008013, MH310740 (Isolate 1a7R-KV03an), MH365349 (Isolate 1a7I-KV08an), and MH365455 (Isolate 1a6R-CH08an).

Sequence homologies of isolates $<98.7 \%$ and $<95 \%$ to the phylogenetically closest type species in the NCBI and Ribosomal Database Project (RDP) databases were deemed to represent candidates for new species within the genus or candidates for new genera within the family, respectively [20,21].

Staphylococcaceae and Enterobacterales isolates were subjected to phenotypic antimicrobial susceptibility testing (AST) using the VITEK ${ }^{\circledR} 2$ system with cards AST-P632 and AST-N214, respectively (all bioMérieux). Enterococcus isolates were screened for resistance against vancomycin, teicoplanin, linezolid, ampicillin, and imipenem using disc diffusion (Thermo Fisher Scientific, Wesel, Germany). Clinical breakpoints of the European Committee on Antimicrobial Susceptibility Testing (EUCAST) were applied to evaluate AST results [22].

Methicillin resistance conferring genes mec $A, \operatorname{mec} B, \operatorname{mec} C$, and $m e c D$ were tested for by PCR in staphylococcal isolates that tested positive for cefoxitin resistance, as previously described [23-25]. Isolates that tested negative were additionally tested with GenoType MRSA (Hain Liefscience GmbH, Nehren, Germany). All S. aureus isolates were subjected to spa typing [26]. Based Upon Repeat Pattern analysis was performed using StaphType software v. 2.2.1 (Ridom GmbH, Würzburg, Germany) with parameters "exclude spa types that are shorter than 5 repeats" and "spa types are clustered if costs are less or equal than 11 ".

Extended-spectrum beta-lactamase (ESBL)-production was tested using MASTDISCS ${ }^{\mathrm{TM}}$ ID Extended-Spektrum- $\beta$-Laktamasen (ES $\beta$ L)-Set (CPD10) D67C (MAST Diagnostica, Reinfeld, Germany), carbapenemase production was tested using imipenem and meropenem disc diffusion (both Thermo Fisher Scientific).

The analysis of the association between MRSA carriage and influencing factors was carried out using Fisher's exact test (GraphPad Prism v.5.00, GraphPad Software, La Jolla, CA, USA). Statistical significance to reject the null hypothesis was assumed at $p<0.05$.

Type strain sequences of isolated bacteria were obtained from the SILVA rRNA database project [27]. CLUSTALW was employed for alignment of sequences; phylogenetic inferences were drawn using Kimura 2-parameter models with the maximum-likelihood method, and MEGA software was used to generate phylogenetic trees.

Similarities and differences of the porcine nasal microflora were elucidated using non-parametric multivariate statistical analysis with PRIMER v6 [28,29].

\section{Results}

\subsection{Culturome Composition and Novel Taxa}

In total, 314 microbial species with standing in nomenclature were cultivated and identified by MALDI-TOF MS and 16S rRNA gene sequencing. Additionally, 41 and 10 isolates were recovered that showed 16S rRNA gene sequence similarities below $98.7 \%$ and $95.0 \%$, respectively, to their closest phylogenetic relatives (Supplementary Figures S1-S11), thus representing novel species and higher taxa candidates, respectively.

Of these 365 microbial species, 147 (40.2\%) belonged to the phylum Firmicutes, 112 (30.7\%) to the phylum Proteobacteria, $89(24.4 \%)$ to the phylum Actinobacteria, 16 (4.4\%) to the phylum Bacteroidetes, and one $(0.3 \%)$ to the phylum of Fusobacteria.

On the genus level, the most frequently isolated bacterial taxa were Rothia (54/54 samples; $100 \%$ ), Staphylococcus (52/54 samples, 96.3\%), Corynebacterium (49/54 samples, 90.7\%), Aerococcus and Streptococcus (each $44 / 54$ samples, $81.5 \%$ ). 
The most frequently isolated bacterial species were Rothia nasimurium (54/54 samples; 100\%), Aerococcus viridans (43/54 samples; 79.6\%), Corynebacterium xerosis (38/54 samples; 70.4\%), and Escherichia coli and Staphylococcus epidermidis (each 32/54 samples; 59.3\%) (Figure 1). Staphylococcus haemolyticus was cultured from 28/54 (51.9\%) samples, Streptococcus hyovaginalis from 26/54 (48.1\%) samples, and Streptococcus suis from 23/54 (42.6\%) samples. Staphylococcus aureus was found in 22/54 (40.7\%) of swabs, Lactococcus raffinolactis in 21/54 (38.9\%), and Lactococcus lactis, Staphylococcus cohnii, and Staphylococcus hyicus in 19/54 (35.2\%) each. Leuconostoc citreum and Streptococcus pluranimalium were recovered from $17 / 54(31.5 \%)$ samples each.

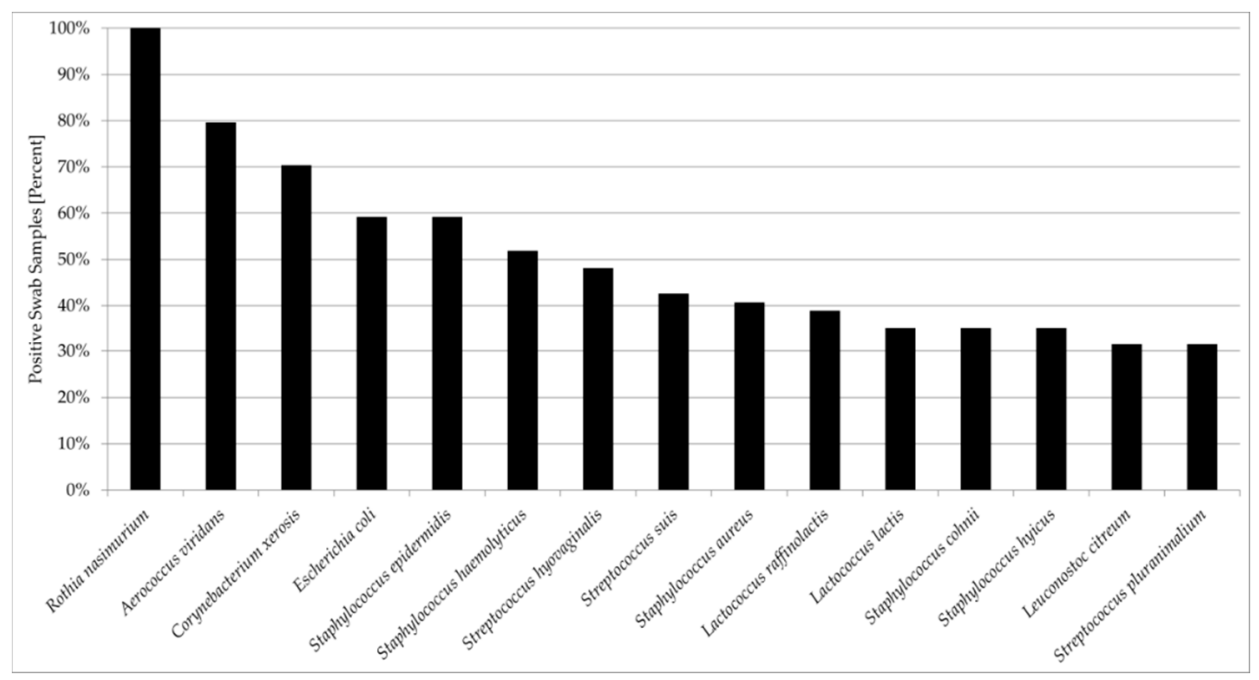

Figure 1. Most commonly isolated bacterial species from 54 porcine nasal swab samples.

\subsubsection{Firmicutes}

Of 147 bacterial taxa belonging to the phylum of Firmicutes, $136(92.5 \%)$ were members of the class of Bacilli, four (2.7\%) of the class of Clostridia, four (2.7\%) of the class of Tissierellia, two (1.4\%) of the class Negativicutes, and one (0.7\%) of the class of Erysipelotrichia.

Of 136 isolates found to be a member of the class Bacilli, 76 (56.3\%) belonged to the order of Lactobacillales and $60(44.1 \%)$ belonged to the order of Bacillales.

Of 60 bacteria belonging to the order Bacillales, 29 (49.2\%) were members of the family Staphylococcaceae (for details, see below), 17 (28.3\%) were members of the family Bacillaceae, nine $(15 \%)$ of the family Planococcaceae, four (6.7\%) of the family Paenibacillaceae, and one (1.7\%) of the family Caryophanaceae. Excepting Staphylococcaceae, Figure S1 shows the phylogenetic relationship of bacteria that were isolated from all samples belonging to these families. Isolates 6a2R-BL08, 6a1R-BL14a, and 4a1I-BL05 had less than 98.7\% 16S rRNA sequence identity to their closest phylogenetic neighbors within the genera of Bacillus, Caryophanon, and Solibacillus, respectively.

Overall, Staphylococcaceae represented $7.9 \%$ of all known and unknown taxa found in this study. They comprised 22 staphylococcal and four macrococcal species. Further species belonged to the genus Jeotgallicoccus $(n=3)$. The phylogenetic relationship of Staphylococcaceae isolated from all samples is given in Figure S2. Of these, isolates 4a1I-BL30 and 3a6R-BL04 exhibited less than 98.7\% 16S rRNA sequence identity to their closest phylogenetic neighbors within the genera of Macrococcus and Jeotgallicoccus, respectively.

At least one pig on each farm was colonized by S. aureus. The spa types of all 51 S. aureus strains that were isolated from all samples were associated with clonal complex (CC) 398 and CC9; the exception is spa type t17059, which has not yet been subjected to multilocus sequence typing or whole genome sequencing. The detected spa types were $\mathrm{t} 899(n=15 ; 29.4 \%), \mathrm{t} 337(n=10 ; 19.6 \%), \mathrm{t} 8893(n=6$; $11.8 \%), \mathrm{t} 034(n=5 ; 9.8 \%), \mathrm{t} 011(n=4 ; 7.8 \%), \mathrm{t} 12359$ and t17059 (each $n=3 ; 5.9 \%), \mathrm{t} 1298$ and t1419 (each 
$n=2 ; 3.9 \%)$, and $2315(n=1 ; 2 \%)$. Figure 2 shows a Based Upon Repeat Pattern (BURP) analysis of the isolated spa types, indicating the long-term evolution of $S$. aureus isolates found in this study.

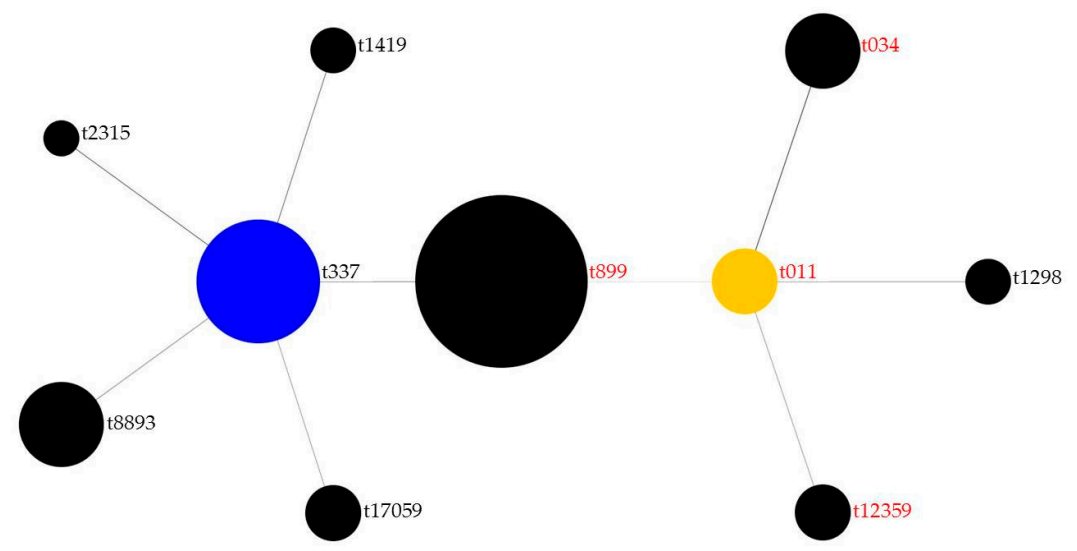

Figure 2. Based Upon Repeat Pattern analysis of 51 isolated spa types. The blue cluster "t 337 " is the founder of ST337, the yellow cluster "t011" is the founder of ST11; spa types in red text were methicillin-resistant Staphylococcus aureus (MRSA). Sizes of dots represent the number of isolates found in this study, connecting lines indicate evolutionary relatedness among spa types.

On all farms, a total of 209 non-S. aureus members of the Staphylococcus genus were isolated. Most frequently, isolates of S. epidermidis $(n=35)$, S. haemolyticus $(n=28)$, S. cohnii $(n=27)$, S. equorum $(n=20)$, and S. hyicus $(n=19)$ were found (Figure 3). Less abundant staphylococcal isolates were S. simulans $(n=14)$, S. pasteuri $(n=13)$, S. chromogenes $(n=11)$, S. sciuri $(n=10)$, and S. hominis $(n=8)$. Additionally, eight other Staphylococcaceae were detected in the course of the investigation: four Macrococcus and four Jeotgallicoccus isolates.

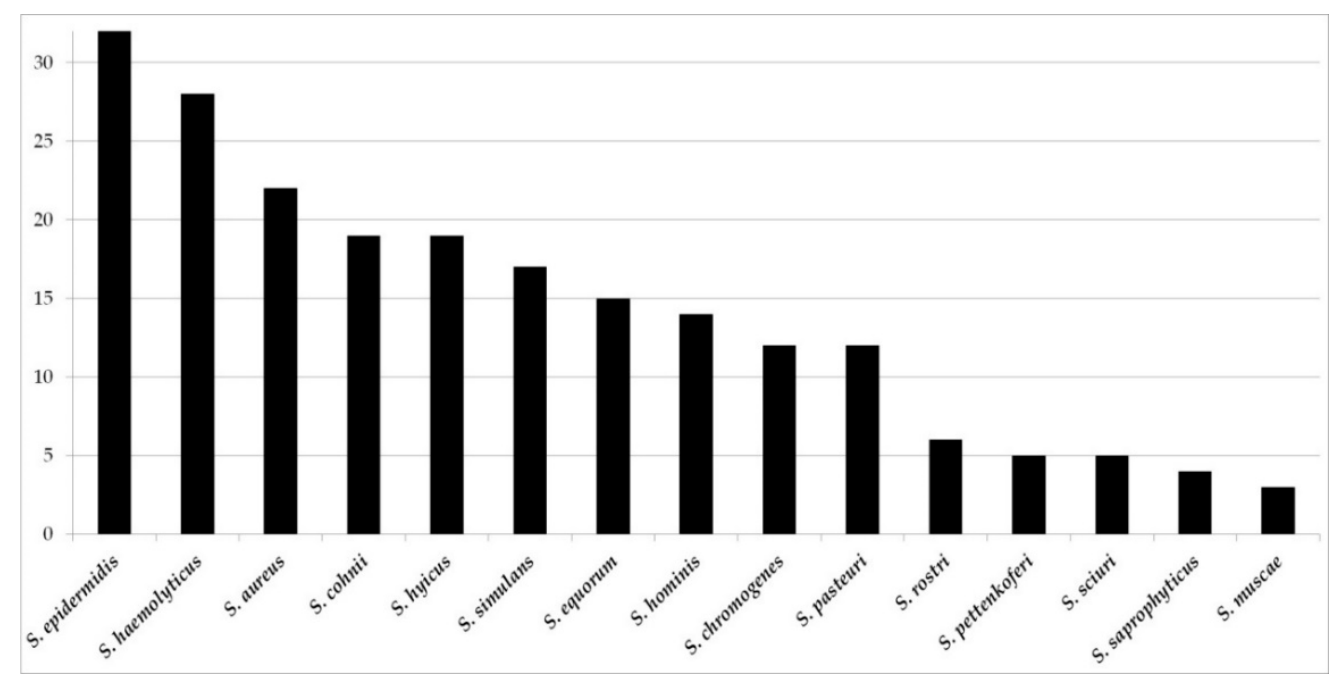

Figure 3. Most commonly isolated staphylococcal species from 54 porcine nasal swab samples.

Of 76 Lactobacillales, $13(17.1 \%)$ were members of the family Enterococcaceae, $10(13.2 \%)$ of the family Aerococcaceae, seven $(9.2 \%)$ of the family Leuconostocaceae, and four (5.3\%) of the family Carnobacteriaceae. Figure S3 shows the phylogenetic relationship of bacteria that were isolated from all samples belonging to these families. Of note, Aerosphaera taetra has yet to be assigned to a family within the order of Lactobacillales. In total, 22 isolates from five out of seven farms belonging to the genus Enterococcus were isolated: five E. eurekensis, three E. aquimarinus and E. devriesei each, two E. faecalis, E. faecium, and novel Enterococcus sp. each, one E. avium, E. gallinarum, E. italicus, E. hirae, and E. casseliflavus each. 
Isolates 1a7I-CH07an, 6a6R-SA02, and 2a1R-BL19 had less than 98.7\% 16S rRNA sequence identity to their closest phylogenetic neighbors within the genera of Enterococcus, Aerococcus, and Globicatella, respectively. Isolates 7a2I-CA08, 4a3R-BL04 (both Facklamia), 6a4I-CA15 (Abiotrophia), 2a1R-CA10 (Globicatella) had less than 95\% 16S rRNA sequence identity to their closest phylogenetic neighbors in the family of Aerococcaceae. Isolates 1a7R-KV03an and 1a7I-KV08an probably lie within the order of Tissierellales with sequence identities below $90 \%$ to their phylogenetic closest matches with standing in nomenclature of Tissierella creatinini and Sporanaerobacter acetigenes, respectively.

Of 76 bacteria belonging to the order Lactobacillales, 22 (28.9\%) were members of the family Streptococcaceae and 18 (23.7\%) were members of the family Lactobacillaceae. Figure S4 shows the phylogenetic relationship of bacteria isolated from all samples belonging to these families. Isolates 6a4I-CA06, 2a1R-BL03, and 6a4R-CH01 had less than 98.7\% 16S rRNA sequence identity to their closest phylogenetic neighbors within the genus of Streptococcus while isolate 4a1I-CA04 was most closely related to Erysipelothrix inopinata (95.55\% sequence identity) and Erysipelothrix rhusiopathiae (95.06\% sequence identity) which belong to the family Erysipelotrichaceae.

\subsubsection{Actinobacteria}

Of 89 bacterial taxa belonging to the phylum and the class of Actinobacteria, 45 (50.6\%) belonged to the order of Micrococcales, 25 (28.1\%) to the order of Corynebacteriales, $17(19.1 \%)$ to the order of Actinomycetales, and one each to the orders Bifidobacteriales and Streptomycetales (1.1\% each).

All 25 isolates belonging to the class of Corynebacteriales belonged to the family of Corynebacteriaceae. Figure S5 shows their phylogenetic relationship. Isolates 1a3R-CA07, 4a1I-BL12, and 4a1R-BL13 had less than $98.7 \%$ 16S rRNA sequence identity to their closest phylogenetic relatives of Corynebacterium vitaeruminis, Corynebacterium humireducens, and Corynebacterium pilosum, respectively. Isolate 7a2R-anCA06 was most closely related to Propioniciclava sinopodophylli with 16S rRNA sequence identity below 95\%. Isolate 1a6I-CH08an probably belonged to the family of Lachnospiraceae, with Faecalicatena fissicatena being its closest phylogenetic relative (93.18\% 16S rRNA sequence identity). Isolate 7a1R-CH28b's closest phylogenetic relation was Helcococcus kunzii.

Of the 45 taxa belonging to the order of Micrococcales, 22 (48.9\%) were members of the family Micrococcaceae and 11 (24.4\%) of the family Microbacteriaceae. Figure S6 shows the phylogenetic relationship of bacteria that were isolated from all samples belonging to these families.

Isolates 7a1I-CH13 and 6a2R-CA11 probably constituted members of the genus Leucobacter and isolate 4a3I-CA14 showed $98.57 \%$ sequence identity to Microbacterium paraoxydans. Isolate 4a1R-BL08 showed most similarities to members of the genus Rothia in its 16S rRNA gene sequence.

Of the 45 taxa belonging to the order of Micrococcales, six $(13.3 \%)$ belonged to the family of Dermabacteraceae, four (8.9\%) to the family of Brevibacteriaceae, and one each (2.2\% each) to the families of Sanguibacteraceae and Promicromonosporaceae. Additionally, the phylogeny of 17 bacteria belonging to the order Actinomycetales with taxa from the families of Propionibacteriaceae $(n=9 ; 52.9 \%)$, Dietziaceae $(n=4 ; 23.6 \%)$, Actinomycetaceae $(n=3 ; 17.6 \%)$, and Nocardiopsaceae $(n=1 ; 5.9 \%)$, and one of each order of Bifidobacteriales and Streptomycetales and their respective families Bifidobacteriaceae and Streptomycetaceae are shown in Figure S7.

Isolate 4a2I-BL24 had less than 98.7\% 16S rRNA sequence identity to its closest phylogenetic neighbor within the genus of Luteococcus. Isolate 7a1I-anCA08 was most closely related to Isoptericola jiangsuensis with a $16 \mathrm{~S}$ rRNA sequence identity of $93.93 \%$. Isolate 4a2I-BL23's closest match was Arcanobacterium phocae (95.82\% sequence similarity).

\subsubsection{Proteobacteria and Bacteroidetes}

Of 112 bacterial taxa belonging to the phylum of Proteobacteria, 86 (76.8\%) belonged to the class of Gammaproteobacteria, $18(16.1 \%)$ to the class of Betaproteobacteria, and eight (7.1\%) to the class of Alphaproteobacteria. 
Of 86 Gammaproteobacteria, 41 (47.7\%) belonged to the order of Pseudomonadales and of these, 24 $(58.5 \%)$ were members of the family Moraxellaceae and 17 (41.5\%) of the family Pseudomonadaceae. Figure S8 shows the phylogenetic relationship of bacteria that were isolated from all samples belonging to these families. Isolates 7a1R-BL14, 1a6I-CH20, 6a2R-MA07, 3a5I-BL02, and 6a1R-BL01 all shared most similarities in their $16 \mathrm{~S}$ rRNA sequences with species of Acinetobacter, namely A. lwoffii, A. piscicola, A. wuhouensis, $A$. junii, and "A. seohaensis", respectively. Isolate 7a2R-BL16 was probably a member of the genus Pseudomonas.

Of all Gammaproteobacteria, eight (9.3\%) were members of the order Aeromonadales and the family Aeromonadaceae, four (4.7\%) of the order Xanthomonadales and the family Xanthomonadaceae, three $(3.5 \%)$ of the order Pasteurellales and the family Pasteurellaceae, and one (1.2\%) of the order Oceanospirillales and the family Oceanospirillaceae; twenty-six (30.2\%) Gammaproteobacteria belonged to the order of Enterobacterales. Of these 26, 16 were members of the family Enterobacteriaceae, four of the family Yersiniaceae, three of the family Erwiniaceae, and three of the family Morganellaceae. Three Gammaproteobacteria are Wohlfahrtiimonas spp. and have yet to be assigned to an order and a family. Figure S9 shows the phylogenetic relationship of the aforementioned bacteria that were isolated from all samples.

Isolate 1a7I-CH24 was a member of the genus Luteimonas. Isolate 5a1I-BL15 was most likely part of the genus Stenotrophomonas. Isolates 5a2R-CA12 and 6a2R-MA08 had the highest percentage identity with Wohlfahrtiimonas populi (97.98\%) and Wohlfahrtiimonas larvae (97.61\%), respectively, and 96.20\% sequence identity to each other.

Of 18 Betaproteobacteria, eight (44.4\%) belonged to the order Neisseriales and the family Neisseriaceae, and $10(55.6 \%)$ to the order Burkholderiales. Of these 10 Burkholderiales, seven (70\%) were members of the family Comamonadaceae and three (30\%) of the family Alcaligenaceae. Of eight Alphaproteobacteria, three $(37.5 \%)$ belonged to the order of Caulobacterales and the family of Caulobacteraceae, and four (50\%) belonged to the order of Rhizobiales. Of these four, three (75\%) were members of the family Brucellaceae and one (25\%) of the family Rhizobiaceae. Enhydrobacter aerosaccus belongs to the order of Rhodospirillales ( $25 \%$ of Alphaproteobacteria) but has yet to be assigned to a family. Figure S10 shows the phylogenetic relationship of the aforementioned bacteria that were isolated from all samples.

Isolate 6a2I-BL10 phylogenetically closest match was Comamonas thiooxydans (98.39\% sequence identity), isolate 1a6R-MA01 was most closely related to Lampropedia puyangensis (97.49\% sequence identity). Isolates 6a6R-CH12 and 1a2R-BL09b showed the highest 16S rRNA sequence similarity to Neisseria shayeganii (96.83\%) and Neisseria dentiae (97.81\%), respectively.

Of 16 bacterial taxa belonging to the phylum of Bacteroidetes, $11(68.8 \%)$ belonged to the class, order and family of Flavobacteriia, Flavobacteriales and Flavobacteriaceae, respectively; three (18.7\%) to the class and order of Bacteroidia and Bacteroidales, respectively, and two (12.5\%) to the class, order and family of Sphingobacteriia, Sphingobacteriales and Sphingobacteriaceae, respectively. Of the three taxa that belonged to the order of Bacteroidales, one (33.3\%) belonged to the family of Rikenellaceae, one $(33.3 \%)$ to the family of Porphyromonadaceae, and one $(33.3 \%)$ has yet to be assigned to a family. Figure S11 shows the phylogenetic relationship of the aforementioned bacteria that were isolated from all samples. Isolate 7a1R-anCH19 may be part of a family within the order of Bacteroidales: Its closest phylogenetic match was Dysgonomonas mossi with $88.67 \%$. Isolate 7a2I-BL11's closest relative on basis of $16 \mathrm{~S}$ rRNA sequence identity was Myroides injenensis (95.91\%) while isolate 4a1I-CH08 probably belonged to the family of Flavobacteriaceae, with Flavobacterium cloacae showing the highest sequence similarity (93.66\%). Isolate 1a4I-BL06a and 4a3I-BL07 were most closely related to species of the genus Chryseobacterium, namely Chryseobacterium haifense (97.66\%) and Chryseobacterium chaponense (96.01\%). Isolate 1a6R-CH08an belonged to the genus of Anaerocella.

\subsection{Comparisons and Correlations of the Porcine Nasal Microbiota}

The similarities of microbiota compositions were determined by non-metric multidimensional scaling plots. Samples from the same farm and of the same sampling date as is indicated by the two 
discernable clusters from farms \#1 and \#6, appear to form clusters, indicating more similarities to each other than to other samples. Samples from farm \#2, however, appear to show more variations in the microflora than other samples (Figure 4).

The porcine nasal microflora is highly diverse: 183 (50.1\%) of 365 bacterial taxa were isolated only once from all samples. Between 13 and 50 taxa were identified per sample, with the lowest mean of 16 from samples of farm \#3 to the highest mean of 38 from samples of farm \#4.

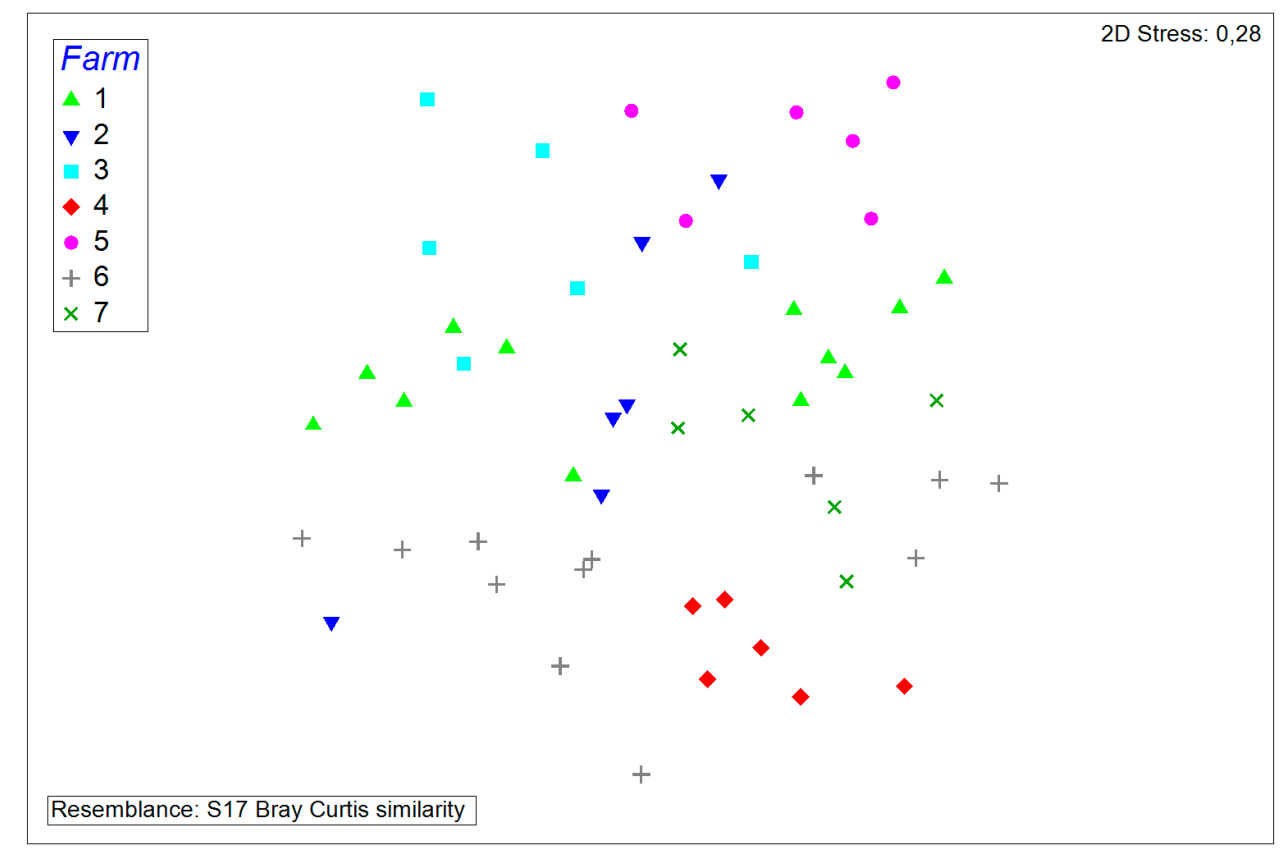

Figure 4. Non-metric multidimensional scaling plot of all samples. Samples from the same farms are represented by the same symbols.

The specific habitat analyzed, i.e., the nasal cavity and surface of the pig snout, respectively, did not seem to play a major role in the composition of the porcine nasal microflora as no distinct clustering is observable in Figure 5. The mean of bacterial taxa isolated from intranasal samples was 24 , the mean of snout surface samples was 26. The lowest number of taxa isolated from intranasal and snout surface swabs was 13 and 14, respectively; the highest amount was 50 and 46, respectively. This indicates similar variability among habitats.

A prominent factor for similarities in samples appears to be the individual itself, i.e., the swab samples from the nasal cavity and the snout surface of the same individual are on the whole relatively similar (Figure 6). However, there are samples of the same individual that are more similar to samples from two different individuals.

Significant positively correlated co-colonization patterns of MRSA vs. age, MRSA vs. S. hyovaginalis (both $p<0.01)$, and MRSA vs. Streptococcus parauberis $(p<0.05)$ by Fisher's exact test (Figure 7a-c). Colonization with Citrobacter sp. (Citrobacter braakii, Citrobacter freundii, Citrobacter gillenii) was statistically significant in its negative correlation with MRSA carriage $(p<0.05$; Figure 7d). Lactococcus lactis narrowly failed to reach statistical significance for a negative correlation $(p=0.074)$. No correlations between the farm factors total number of pigs, presence of other animals, farm type, and feed type were found. 


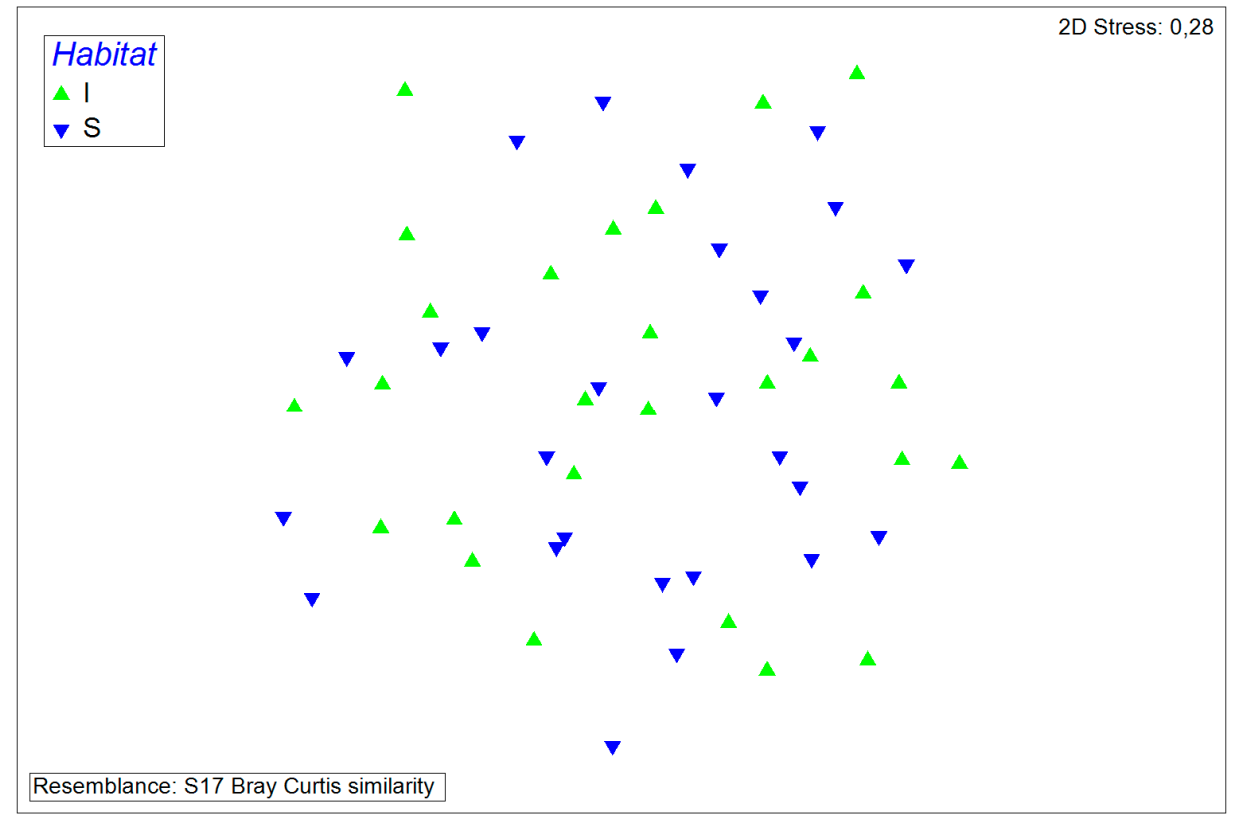

Figure 5. Non-metric multidimensional scaling plot of all samples. Samples from the same habitat are represented by the same symbols. "I" denotes intranasal swab samples, "S" denotes snout surface samples.

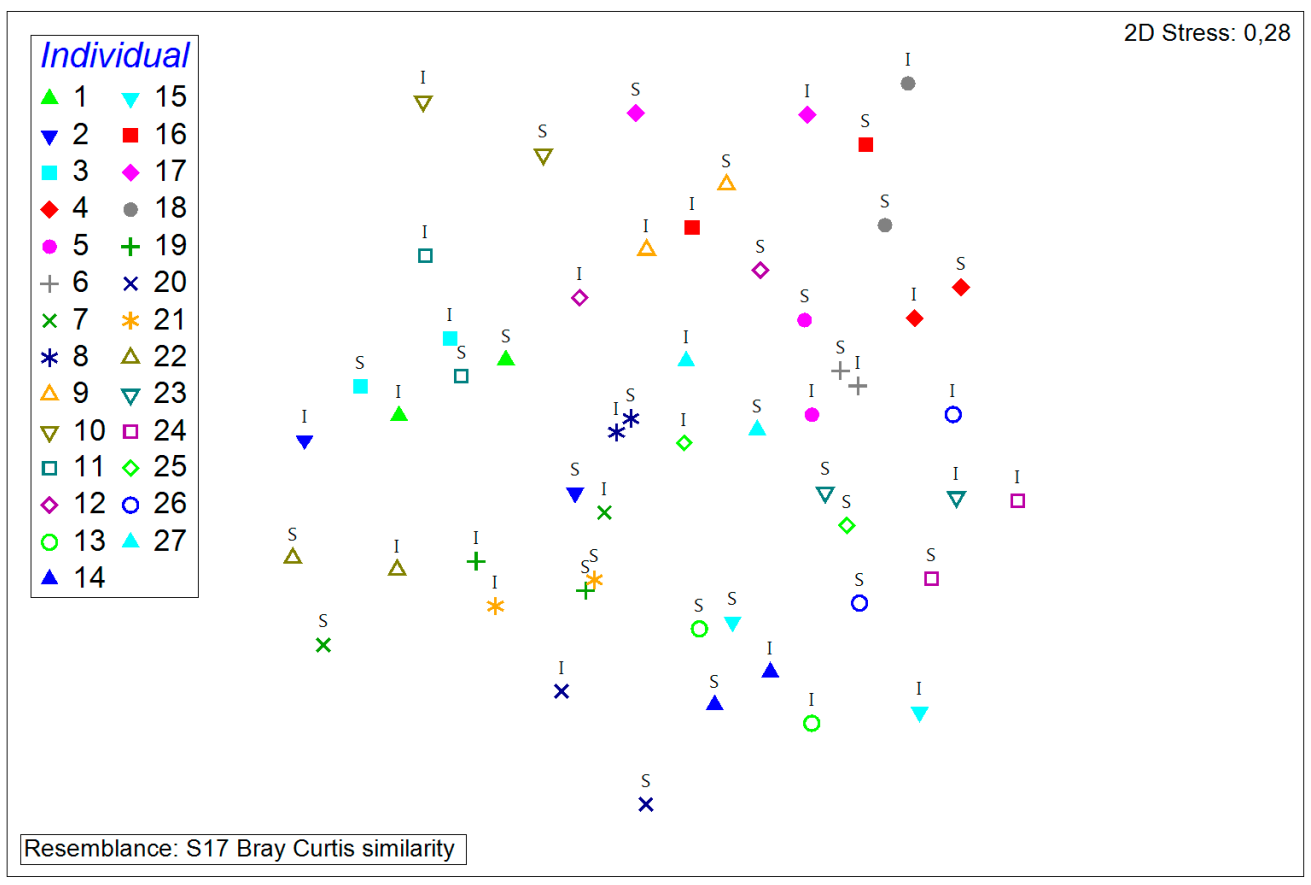

Figure 6. Non-metric multidimensional scaling plot of all samples. Samples from the same individual are represented by the same symbols. "I" denotes intranasal swab samples, "S" denotes snout surface samples. 

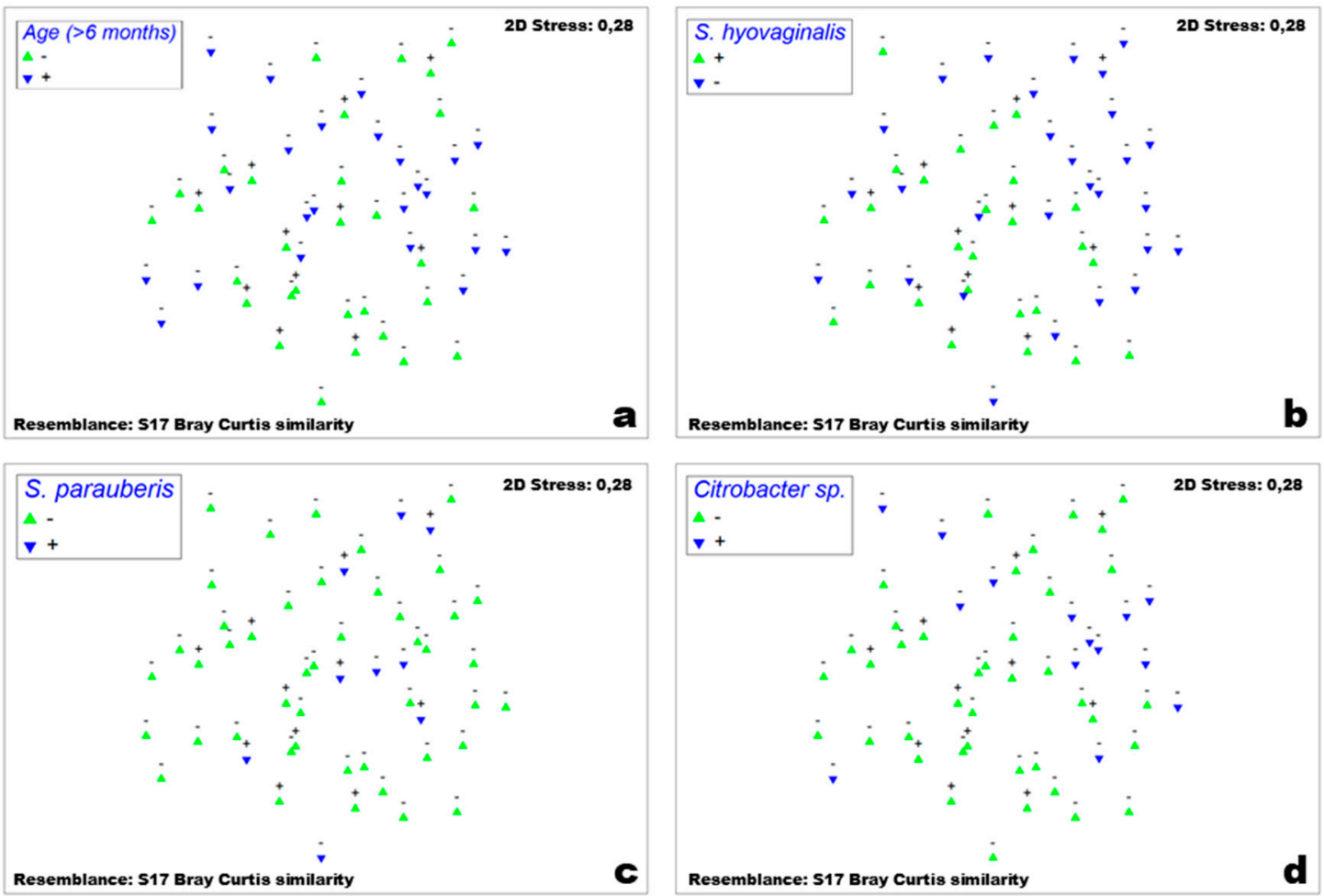

Figure 7. Non-metric multidimensional scaling plot of all samples. MRSA-positive samples are marked with "+", MRSA-negative samples are marked with "-" above the symbols. (a) Green triangles represent samples of pigs that were six months of age or younger, blue inverted triangles represent samples of pigs that were older than six months on sampling date. (b) Green triangles represent samples of pigs that were positive for $S$. hyovaginalis colonization, blue inverted triangles represent samples of pigs that were negative for $S$. hyovaginalis colonization on sampling date. (c) Green triangles represent samples of pigs that were negative for S. parauberis colonization, blue inverted triangles represent samples of pigs that were positive for S. parauberis colonization on sampling date. (d) Green triangles represent samples of pigs that were positive for Citrobacter sp. colonization, blue inverted triangles represent samples of pigs that were negative for Citrobacter sp. colonization on sampling date.

\subsection{Analysis of Resistance}

S. aureus isolates exhibiting resistance towards methicillin $(n=26 ; 51 \%)$ were detected on five out of seven farms (85.7\%), all of which tested positive for mecA (Table 2). MRSA spa types were $\mathrm{t} 011, \mathrm{t} 034$, $\mathrm{t} 899$, and $\mathrm{t} 12359$ (Figure 2); one isolate with spa type $\mathrm{t} 899$ did not exhibit methicillin resistance or test positive for $m e c A$.

Other resistances included $26(51 \%)$ tetracycline-resistant isolates on four out of seven farms (57.1\%), 10 (19.6\%) clindamycin-resistant isolates on four out of seven farms (57.1\%), seven (13.7\%) trimethoprim/sulfamethoxazole-resistant isolates on four out of seven farms (57.1\%), eight (15.7\%) erythromycin-resistant isolates on three out of seven farms (42.8\%), and 19 (37.2\%) levofloxacin-resistant isolates from two out of seven farms $(28.6 \%)$.

Phenotypic methicillin resistance with detectable mec resistance gene was detected in 23 Staphylococcus isolates (11.0\%; all mecA) other than S. aureus on three out of seven farms (42.9\%) (Table 3). Isolates resistant to benzylpenicillin $(n=66 ; 31.6 \%)$, clindamycin $(n=78 ; 37.3 \%)$, erythromycin $(n=68$; $32.5 \%)$, fosfomycin $(n=90 ; 43.1 \%)$, fusidic acid $(n=51 ; 24.4 \%)$, and tetracycline $(n=101 ; 48.3 \%)$ were found on seven out of seven farms, while trimethoprim/sulfamethoxazole resistance was prevalent in seven isolates from four out of seven farms. Two Macrococcus goetzii isolates exhibited methicillin resistance and tested positive for $m e c B$. 
Table 2. S. aureus isolates with spa types, detected mecA genes, and phenotypic antimicrobial susceptibility test results.

\begin{tabular}{|c|c|c|c|c|c|c|c|}
\hline \multirow{2}{*}{ Farm } & \multirow{2}{*}{ Individual } & \multirow{2}{*}{$\underset{\text { Site }}{\text { Sampling }}$} & \multirow{2}{*}{ spa Type } & \multirow[t]{2}{*}{$\begin{array}{l}\text { Resistance } \\
\text { Gene } m e c A\end{array}$} & \multicolumn{2}{|c|}{$\begin{array}{l}\text { Phenotypic Antimicrobial Susceptibility } \\
\text { Test Profile a }^{{ }^{a}}\end{array}$} & \multirow[t]{2}{*}{$\begin{array}{c}\# \text { of } \\
\text { Isolates }\end{array}$} \\
\hline & & & & & $\begin{array}{c}\text { CXI } \\
\text { Screening }\end{array}$ & Other Resistances & \\
\hline \multirow{10}{*}{$\# 1$} & \multirow{2}{*}{$\# 1$} & $\begin{array}{l}\text { Nasal } \\
\text { cavity }\end{array}$ & t011 & + & + & OXA, BEN, TET & 2 \\
\hline & & $\begin{array}{l}\text { Snout } \\
\text { surface }\end{array}$ & t011 & + & + & OXA, BEN, TET & 1 \\
\hline & \multirow{4}{*}{$\# 2$} & \multirow{2}{*}{$\begin{array}{l}\text { Nasal } \\
\text { cavity }\end{array}$} & $\mathrm{t} 1298$ & $\mathrm{n} / \mathrm{a}$ & - & BEN, TET & 2 \\
\hline & & & $\mathrm{t} 337$ & $\mathrm{n} / \mathrm{a}$ & - & BEN, TET & 1 \\
\hline & & \multirow{2}{*}{$\begin{array}{l}\text { Snout } \\
\text { surface }\end{array}$} & t011 & + & + & OXA, BEN, TET & 1 \\
\hline & & & $\mathrm{t} 337$ & $\mathrm{n} / \mathrm{a}$ & - & BEN, TET & 2 \\
\hline & & $\begin{array}{l}\text { Nasal } \\
\text { cavity }\end{array}$ & $\mathrm{t} 337$ & $\mathrm{n} / \mathrm{a}$ & - & BEN, TET & 1 \\
\hline & \#3 & & t8893 & $\mathrm{n} / \mathrm{a}$ & - & BEN, TET & 1 \\
\hline & & $\begin{array}{l}\text { snout } \\
\text { surface }\end{array}$ & $\mathrm{t} 337$ & $\mathrm{n} / \mathrm{a}$ & - & BEN, TET & 4 \\
\hline & & & t1419 & $\mathrm{n} / \mathrm{a}$ & - & BEN, TET, TRS & 2 \\
\hline \multirow{3}{*}{$\# 2$} & \multirow{3}{*}{$\# 2$} & \multirow{2}{*}{$\begin{array}{l}\text { Nasal } \\
\text { cavity }\end{array}$} & t337 & $\mathrm{n} / \mathrm{a}$ & - & BEN & 2 \\
\hline & & & t8893 & $\mathrm{n} / \mathrm{a}$ & - & BEN & 2 \\
\hline & & $\begin{array}{l}\text { Snout } \\
\text { surface }\end{array}$ & t8893 & $\mathrm{n} / \mathrm{a}$ & - & BEN & 2 \\
\hline \multirow{2}{*}{ \#3 } & $\# 1$ & $\begin{array}{l}\text { Snout } \\
\text { surface }\end{array}$ & t17059 & $\mathrm{n} / \mathrm{a}$ & - & LEV, BEN & 1 \\
\hline & $\# 2$ & $\begin{array}{c}\text { Snout } \\
\text { surface }\end{array}$ & t17059 & $\mathrm{n} / \mathrm{a}$ & - & LEV, BEN & 2 \\
\hline \multirow{2}{*}{$\# 4$} & $\# 1$ & $\begin{array}{l}\text { Snout } \\
\text { surface }\end{array}$ & t8893 & $\mathrm{n} / \mathrm{a}$ & - & BEN & 1 \\
\hline & $\# 2$ & $\begin{array}{c}\text { Snout } \\
\text { surface }\end{array}$ & t034 & + & + & CLI, ERY, BEN, TRS, TET & 1 \\
\hline \multirow{3}{*}{ \#5 } & \multirow{3}{*}{$\# 1$} & \multirow{2}{*}{$\begin{array}{l}\text { Nasal } \\
\text { cavity }\end{array}$} & t034 & + & + & $\begin{array}{l}\text { CLI, ERY, OXA, BEN, } \\
\text { TRS, TET }\end{array}$ & 2 \\
\hline & & & t034 & + & + & CLI, ERY, OXA, BEN, TET & 1 \\
\hline & & $\begin{array}{l}\text { Snout } \\
\text { surface }\end{array}$ & t034 & + & + & $\begin{array}{l}\text { CLI, ERY, OXA, BEN, } \\
\text { TRS, TET }\end{array}$ & 1 \\
\hline \multirow{6}{*}{ \#6 } & $\# 2$ & $\begin{array}{l}\text { Nasal } \\
\text { cavity }\end{array}$ & t899 & + & + & CLI, LEV, OXA, BEN & 1 \\
\hline & \multirow{4}{*}{ \#3 } & \multirow{3}{*}{$\begin{array}{l}\text { Nasal } \\
\text { cavity }\end{array}$} & t899 & $\mathrm{n} / \mathrm{a}$ & - & LEV, BEN & 1 \\
\hline & & & t899 & + & + & LEV, OXA, BEN & 3 \\
\hline & & & t899 & + & + & CLI, LEV, OXA, BEN & 1 \\
\hline & & $\begin{array}{l}\text { Snout } \\
\text { surface }\end{array}$ & t899 & + & + & LEV, OXA, BEN & 9 \\
\hline & $\# 4$ & $\begin{array}{l}\text { Snout } \\
\text { surface }\end{array}$ & $\mathrm{t} 2315$ & $\mathrm{n} / \mathrm{a}$ & - & BEN & 1 \\
\hline \multirow{2}{*}{$\# 7$} & \multirow{2}{*}{$\# 1$} & $\begin{array}{l}\text { Nasal } \\
\text { cavity }\end{array}$ & t12359 & + & + & $\begin{array}{l}\text { CLI, ERY, OXA, BEN, } \\
\text { TRS, TET }\end{array}$ & 1 \\
\hline & & $\begin{array}{l}\text { Snout } \\
\text { surface }\end{array}$ & t12359 & + & + & CLI, ERY, OXA, BEN, TET & 2 \\
\hline
\end{tabular}

+, positive; - , negative; n/a, not applicable. ${ }^{a}$ as determined by Vitek 2 using cards AST-P632; BEN, benzylpenicillin; CLI, clindamycin; CXI, cefoxitin; ERY, erythromycin; LEV, levofloxacin; OXA, oxacillin; TET, tetracycline; TRS, trimethoprim/sulfamethoxazole. 
Table 3. Oxacillin-resistant non-S. aureus staphylococci including two Macrococcus goetzii isolates with detected $m e c A$ genes and phenotypic antimicrobial susceptibility test results.

\begin{tabular}{|c|c|c|c|c|c|}
\hline Farm & Individual & Sampling Site & Species & Resistance Gene & Resistances $^{a}$ \\
\hline \multirow{12}{*}{$\# 4$} & \multirow{6}{*}{$\# 1$} & \multirow{3}{*}{ Nasal cavity } & S. pasteuri & mecA & FOS, OXA, BEN, TET \\
\hline & & & S. haemolyticus & mec $A$ & CLI, FOS, GEN, CXI, BEN, TET \\
\hline & & & S. pasteuri & mec $A$ & FOS, OXA, BEN, TET \\
\hline & & \multirow{3}{*}{ Snout surface } & S. hominis & mecA & FOS, OXA, BEN, TET \\
\hline & & & S. pasteuri & mecA & CLI, FOS, OXA, CXI, BEN \\
\hline & & & S. pasteuri & mec $A$ & CLI, FOS, OXA, CXI, BEN \\
\hline & \multirow{5}{*}{$\# 2$} & \multirow{3}{*}{ Nasal cavity } & S. saprophyticus & mecA & FOS, OXA, CXI, BEN, TET \\
\hline & & & S. pasteuri & mec $A$ & FOS, OXA, BEN, TET \\
\hline & & & S. epidermidis & mecA & CLI, OXA, CXI, TET \\
\hline & & \multirow{2}{*}{ Snout surface } & S. haemolyticus & mecA & CLI, FOS, GEN, CXI, BEN, TET \\
\hline & & & S. cohnii & mec $A$ & $\begin{array}{c}\text { CLI, ERY, FUS, OXA, CXI, BEN, } \\
\text { TRS, TET }\end{array}$ \\
\hline & $\# 3$ & Nasal cavity & S. pasteuri & mec $A$ & FOS, OXA, CXI, BEN, TET \\
\hline \multirow{11}{*}{$\# 5$} & \multirow{6}{*}{$\# 1$} & \multirow{3}{*}{ Nasal cavity } & S. sciuri & mecA & CLI, DAP, FUS, OXA, CXI \\
\hline & & & S. sciuri & mec $A$ & CLI, DAP, FUS, OXA, BEN, TET \\
\hline & & & M. goetzii & mecB & CLI, ERY, FOS, OXA, TET \\
\hline & & \multirow{3}{*}{ Snout surface } & M. goetzii & mecB & CLI, ERY, FOS, OXA, CXI, TET \\
\hline & & & S. epidermidis & mec $A$ & $\begin{array}{c}\text { CLI, ERY, FOS, OXA, CXI, BEN, } \\
\text { TET }\end{array}$ \\
\hline & & & S. equorum & mec $A$ & $\begin{array}{c}\text { CLI, ERY, FOS, OXA, CXI, BEN, } \\
\text { TET }\end{array}$ \\
\hline & \multirow{5}{*}{$\# 2$} & \multirow{5}{*}{ Snout surface } & S. epidermidis & mec $A$ & $\begin{array}{c}\text { CLI, ERY, FOS, OXA, CXI, BEN, } \\
\text { TET }\end{array}$ \\
\hline & & & S. sciuri & mec $A$ & $\begin{array}{l}\text { CLI, DAP, ERY, FUS, OXA, CXI, } \\
\text { BEN, TET }\end{array}$ \\
\hline & & & S. sciuri & mec $A$ & $\begin{array}{c}\text { CLI, DAP, ERY, FUS, OXA, CXI, } \\
\text { BEN, TET }\end{array}$ \\
\hline & & & S. sciuri & mec $A$ & $\begin{array}{c}\text { CLI, DAP, ERY, FUS, OXA, CXI, } \\
\text { BEN, TET }\end{array}$ \\
\hline & & & S. haemolyticus & $\operatorname{mec} A$ & $\begin{array}{c}\text { CLI, ERY, FOS, GEN, OXA, CXI, } \\
\text { BEN, TET }\end{array}$ \\
\hline \multirow{2}{*}{$\# 7$} & $\# 2$ & Snout surface & S. pasteuri & mec $A$ & $\begin{array}{c}\text { CLI, ERY, FOS, OXA, CXI, BEN, } \\
\text { TET }\end{array}$ \\
\hline & \#3 & Snout surface & S. pasteuri & mecA & CLI, ERY, FOS, OXA, BEN, TET \\
\hline
\end{tabular}

${ }^{a}$ as determined by Vitek 2 using cards AST-P632; BEN, benzylpenicillin; CLI, clindamycin; CXI, cefoxitin; ERY, erythromycin; FOS, fosfomycin; FUS, fusidic acid; LEV, levofloxacin; OXA, oxacillin; TET, tetracycline; TRS, trimethoprim/sulfamethoxazole.

Screening for resistance to vancomycin, teicoplanin, linezolid, ampicillin, and imipenem in 22 Enterococcus isolates yielded one vancomycin-resistant E. casseliflavus, two ampicillin- and imipenem-resistant $E$. faecium, and one ampicillin-resistant $E$. avium.

None of the 106 E. coli isolates from 7/7 farms and none of the 19 Klebsiella isolates from five out of seven farms tested positive for either ESBL production or carbapenemase production.

Resistance to ampicillin was detected in $48 / 106(45.3 \%)$ E. coli isolates on six out of seven farms $(85.7 \%)$ (Tables S1 and S2). Of these 48 isolates, $42(87.5 \%)$ were isolates from farms \#1 $(n=10 ; 20.8 \%)$, $\# 3(n=17 ; 35.4 \%)$, and \#5 $(n=15 ; 31.3 \%)$, while farm \#2 harbored four $(8.3 \%)$ isolates and farms \#4 and $\# 6$ harbored one $(2.1 \%)$ isolate each. Farm \#7 tested negative for the presence of ampicillin-resistant $E$. coli. 


\section{Discussion}

In this study, we used a qualitative, extensive culturomics approach to examine the microbial porcine nasal flora. This in-depth approach resulted in the isolation of 51 hitherto undescribed bacterial taxa according to commonly used 16S rRNA similarity thresholds [20]. This is in contrast to previous publications in which quantitative, molecular methods were employed [30-32].

Lowe and colleagues defined the core microbiota in the tonsils as being dominated by Pasteurellaceae which were found in all twelve animals examined [30]. We have found members of this family but in far lower quantity, with eight of the 27 individuals (nine out of 54 samples) carrying Pasteurella aerogenes, P. multocida, or Haemophilus parasuis. Although a statistically significant correlation $(p<0.05)$ is reached for Pasteurellaceae carriage and age ( $\leq 24$ weeks and $>24$ weeks), our data indicate that bacteria of this family are less likely to be found in older pigs (11 non-carriers vs. one carrier) than younger pigs (seven non-carriers vs. eight carriers). Additionally, the phylum of Proteobacteria and the genus Streptococcus mark similarities between our findings as these were isolated from all and almost all (26/27) of the sampled individuals in our study and in all of the tonsils tested by Lowe and colleagues. However, the genus Actinobacillus, which was highly abundant in most samples in the pyrosequencing approach, could not be isolated at all from our samples. Further discrepancies include detection of the genera Alkanindiges, Peptostreptococcus, Veillonella, and Fusobacterium which Lowe et al. detected in all samples, but we did not find at all (Alkanindiges, Peptostreptococcus) or rarely (Veillonella: two out of 27 individuals, two out of 54 samples; Fusobacterium: one out of 27 individuals, two out of 54 samples). In the same study, Neisseriaceae were detected in all samples; we found bacteria belonging to this genus in 13/27 animals (16/54 samples). These differences might stem from multiple reasons, be it sampling site, method used or antibiotic treatment. In the study of Lowe, tylosin, a macrolide approved only for veterinarian medicine, was administered to four out of 12 pigs in-feed, another four out of 12 pigs received additional tilmicosin, another veterinary macrolide [30]. The influence of the administered macrolides, which inhibit the growth of a broad range of Gram-positive bacteria and show less activity against Gram-negatives, may partly explain the discrepancies we found.

Weese and colleagues conducted a study in which 13/20 sampled pigs also received tylosin, but they evaluated the nasal microbiota rather than tonsillar microbiota [31]. While the dominant phylum in their data set was Proteobacteria as well, they reported different predominant genera: Moraxella, Psychrobacter, and Pseudomonas make up $71.4 \%$ of the relative abundance. We isolated bacteria belonging to these genera from 23/27 individuals (37/54 samples), indicating a high prevalence detectable by culturomics as well. However, the genera of Acinetobacter and Aerococcus which accounted for $4.8 \%$ and $1.8 \%$ of the relative abundance in the aforementioned publication, respectively, were isolated from 18/27 individuals (25/54 samples) and 26/27 individuals (44/54 samples), respectively. Despite varying overall results, the observation that two operational taxon units (OTUs) of species of lactic-acid bacteria indicate MRSA-negative pigs is to some degree similar to our finding that Lactococcus colonization trends toward a negative correlation with MRSA isolation. The conclusion of the authors that Staphylococcus was found to be an indicator OTU for MRSA-negative pigs cannot be confirmed by our results because we have found staphylococci in all individuals and almost all samples (52/54) [31]. Considering that coagulase-negative staphylococci (CoNS) from human and animal sources may harbor mec genes as part of mobile SCCmec elements and therefore may act as a reservoir for methicillin resistance, experimental approaches to conquer LA-MRSA in pigs by competitive colonization with CoNS are questionable [9,33-35].

The most recent study investigating the porcine nose microbiome by Strube et al. showed some differences compared to the two aforementioned studies and most similarities to our findings: Rothia, Streptococcus, Aerococcus, and Staphylococcus were found in all samples of the nasal cavity with varying abundance [32]. However, the staphylococcal species they found are differently distributed. Staphylococcus equorum dominated the genus of Staphylococcus in all samples analyzed using the tuf-gene approach, while we isolated this species from 11/27 individuals (15/54 samples). On the other hand, the most frequently isolated staphylococci in our culturomics approach were S. epidermidis (24/27 
individuals; 32/54 samples) and S. haemolyticus (21/27 individuals; 28/54 samples). Of note, Strube and colleagues concede that the Staphylococcus specific primers positively biased S. equorum and very negatively biased S. epidermidis and S. haemolyticus. One of their results that is contrary to our data is that Streptococcus is "inversely associated with Staphylococcus and MRSA" [32]; we have shown a positive correlation between isolation of MRSA and Streptococcus hyovaginalis and Streptococcus parauberis. Furthermore, samples from which three or more streptococcal species were isolated were more likely to be positive for S. aureus and MRSA colonization. This may be due to entirely opposite biases in both techniques and it stresses that a combination of culture-based and molecular approaches is needed to study the microbiota composition and the interactions within as already shown for respective analyses of human microbiota [36]. It is also possible that there are species within the large and diverse genus of Streptococcus that either exhibit synergistic or antagonistic effects on $S$. aureus colonization. Both S. hyovaginalis and S. parauberis are associated with pigs and cows, respectively, and can therefore probably be found in samples from farms. Colonization with Citrobacter spp. is also common, albeit as carriers and reservoirs for resistance genes; intervention strategies employing species of this genus should be considered very carefully as this may only cause the substitution of one threat with another. Why and how the aforementioned bacterial taxa can possibly affect MRSA colonization need to be elucidated in further studies. Aside from microbial colonization, we have found a correlation between pigs older than six months and MRSA carriage. This result was indirectly supported by a study that found farm workers six times more likely to be colonized with MRSA if they worked with farrowing, i.e., older sows [37]. Whether this observation is e.g., merely the outcome of longer exposure to MRSA and therefore higher cumulative risk of carriage remains unknown; future research may shed light on this phenomenon.

All in all, differences in results are most likely due to methodological biases, antibiotic exposure, and geographic location: The studies by Lowe, Weese, and Strube were conducted in the United States, Canada, and Denmark, respectively. A potential role of bacteriocins should be further investigated as indications are given here and in the publications cited above that certain lactic-acid bacteria are inversely correlated with S. aureus and MRSA colonization [38]. Three years ago, a compound produced by the human nose colonizer $S$. lugdunensis called lugdunin has been isolated and shown to inhibit growth of $S$. aureus [39]. There is the possibility of microorganisms producing antimicrobials in the porcine nasal cavity as well due to relatively high colonization pressure. Considering the amount of potentially new bacterial taxa with largely unknown metabolic properties isolated by our approach, similar defense mechanisms are possible. The strain collection we have obtained from our samples will be useful in examining these hypotheses.

Seven of 27 sampled pigs (25.9\%) tested positive for MRSA colonization. This is in agreement with previous studies [40]. Different spa types on the same farm were detected on four out of seven farms, different spa types from the same sample, i.e., habitats were detected in four out of 20 samples positive for S. aureus. Oxacillin resistance is also not evenly distributed among isolates from the same farm and in two cases not even from the same habitat. The co-colonization of isolates with different spa types and resistances towards oxacillin, i.e., methicillin-susceptible S. aureus and MRSA, has previously been described by Fetsch and colleagues; our data support their findings [41].

All levofloxacin-resistant S. aureus isolates were MRSA strains with the exception of those from farm \#3. Additionally, the only CoNS isolate exhibiting levofloxacin resistance was an S. hyicus from the same farm. This is probably in direct correlation with the enrofloxacin treatment received by two of the three sampled pigs at some time point in the previous three months. Regarding resistance patterns of CoNS, we found worrying data: Schoenfelder and colleagues reported varying antibiotic resistance detection in dust and manure samples among 41 pig farms. Tetracycline was the only class to which resistance had been detected on all farms [42]; we have found CoNS resistant to tetracycline, benzylpenicillin (beta-lactam), clindamycin (lincosamide), erythromycin (macrolide), fosfomycin, and fusidic acid on all farms tested. Furthermore, we isolated at least two different CoNS species resistant towards three or more different antibiotic classes from all farms, i.e., farm \#1 harbored a Staphylococcus 
rostri isolate resistant towards clindamycin, fosfomycin, and tetracycline, as well as a Staphylococcus $x y l o s u s$ isolate resistant to the same three antibiotics and fusidic acid. Nine of eleven Staphylococcus sciuri isolates from four out of seven farms exhibited daptomycin resistance using Vitek. Etests further indicated lowered susceptibility in seven out of nine isolates. This is alarming because the cyclic lipopeptide daptomycin is a key drug for the treatment of severe $S$. aureus infections and diminished susceptibility in CoNS has been reported on pig farms before [42]. Discrepancies in results may again stem from the type of sample used. Schoenfelder and colleagues collected environmental samples from dust and manure. It is possible that this difference accounts for the incongruous findings.

We found no evidence of ESBL-producing or carbapenem-resistant Enterobacteriaceae; ampicillin resistance was detected on six out of seven farms, although with vast differences in the number of isolates. One explanation for this finding is the habitat and sampling approach: studies investigating ESBL-E or carbapenemase-producing Enterobacteriaceae examined fecal or environmental samples that were pooled, increasing the probability to detect the bacteria in question [43]. Of note, we found the vast majority (20/21 isolates) of fluoroquinolone-resistant $E$. coli in samples from two individuals that were treated with enrofloxacin within three months for five days prior to sampling. Similarly, all E. coli isolates from farm \#5 show resistance towards beta-lactam agents (15/48). Although less pronounced in this case because ampicillin resistance is frequently found on other farms, this was the only farm where not one isolate was found to be susceptible to a beta-lactam agent. Many factors confer quinolone resistance, at times causing resistance towards other antimicrobial agents as well [44]. These findings further underpin the vast body of evidence highlighting the importance of antibiotic stewardship in veterinary medicine that is at least in Germany starting to take effect $[45,46]$.

\section{Conclusions}

Our approach sought to reconstruct the cultivable constituents of the porcine nasal microflora as closely as possible to natural colonization patterns. The study at hand has found Rothia nasimurium to be a main constituent of the porcine nasal microflora. Multiple bacterial isolates appear to constitute hitherto undescribed taxa. This fact can pose an untapped potential or unknown threat because roles as both, producer of unknown antimicrobial agents or resistance gene reservoirs are possible. The frequency of MRSA on farms is comparable to previous reports. Antibiotic resistance gene carriage by CoNS and macrococci must not be ignored and should be monitored. No ESBL-producing E. coli or Klebsiella isolates were found, possibly due to the examined habitat. It is of paramount importance to combat this threat to animal and human health care, and therefore to implement "One Health" approaches.

Supplementary Materials: The following are available online at http://www.mdpi.com/2076-2607/8/4/514/s1, Figure S1: Position of isolates 4a1I-BL05, 6a1R-BL14a, and 6a2R-BL08 in phylogenetic relation to closely related bacterial species also isolated from porcine nasal swab samples, Figure S2: Position of isolates 4a1I-BL30 and 3a6R-BL04 in phylogenetic relation to closely related bacterial species also isolated from porcine nasal swab samples, Figure S3: Position of isolates 1a7I-CH07an, 6a4I-CA15, 7a2I-CA08, 4a3R-BL04, 2a1R-CA10, 2a1R-BL19, 6a6R-SA02, 4a1I-CA04, 1a6I-CH08an, 1a7R-KV03an, 1a7I-KV08an, and 7a1R-CH28b in phylogenetic relation to closely related bacterial species also isolated from porcine nasal swab samples, Figure S4: Position of isolates 2a1R-BL03, 6a4I-CA06, and 6a4R-CH01 in phylogenetic relation to closely related bacterial species also isolated from porcine nasal swab samples, Figure S5: Position of isolates 4a1I-BL12, 1a3R-CA07, and 4a1R-BL13 in phylogenetic relation to closely related bacterial species also isolated from porcine nasal swab samples, Figure S6: Position of isolates 4a1R-BL08, 7a1I-CH13, 6a2R-CA11, and 4a3I-CA14, in phylogenetic relation to closely related bacterial species also isolated from porcine nasal swab samples, Figure S7: Position of isolates 7a1I-anCA08, 4a2I-BL23, 7a2R-anCA06, and 4a2I-BL24 in phylogenetic relation to closely related bacterial species also isolated from porcine nasal swab samples, Figure S8: Position of isolates 7a1R-BL14, 1a6I-CH20, 6a2R-MA07, 6a1R-BL01a, 3a5I-BL02, and 7a2R-BL16 in phylogenetic relation to closely related bacterial species also isolated from porcine nasal swab samples, Figure S9: Position of isolates 5a2R-CA12, 6a2R-MA08, 1a7I-CH24, and 5a1I-BL15 in phylogenetic relation to closely related bacterial species also isolated from porcine nasal swab samples, Figure S10: Position of isolates 6a2I-BL10, 1a6R-MA01, 1a2R-BL09b, and 6a6R-CH12, in phylogenetic relation to closely related bacterial species also isolated from porcine nasal swab samples, Figure S11: Position of isolates 4a3I-BL07, 1a4I-BL06a, 4a1I-CH08, 7a2I-BL11, 7a1R-anCH19, and 1a6R-CH08an in phylogenetic relation to closely related bacterial species also isolated from porcine nasal swab samples, Table S1: Phenotypic antimicrobial susceptibility 
profiles of $E$. coli isolates from farms \#1 to \#3, Table S2: Phenotypic antimicrobial susceptibility profiles of $E$. coli isolates from farms \#4 to \#7.

Author Contributions: Conceptualization, U.K. and K.B.; Data curation, A.S. and U.K.; Formal analysis, A.S.; Funding acquisition, K.B.; Investigation, A.S.; Methodology, A.S., K.v.L. and U.K.; Project administration, U.K. and K.B.; Resources, K.v.L.; Supervision, K.B.; Validation, U.K. and K.B.; Writing-original draft, A.S.; Writing-review \& editing, K.v.L., U.K. and K.B. All authors have read and agreed to the published version of the manuscript.

Funding: This research was funded by the German Federal Ministry of Education and Research (Bundesministerium für Bildung und Forschung, BMBF) within the research consortia InfectControl 2020 and \#1Health-PREVENT (grant no. 03ZZ0802H and 01KI1727A to KB), and in part by the Federal Institute for Risk Assessment (Bundesinstitut für Risikobewertung, BfR) (grant no. 1329-557 to KB).

Acknowledgments: This work is dedicated to the memory of Georg Peters (1951-2018). We thank Annette Feldkamp, Martina Schulte, and Anja Hassing for excellent technical assistance.

Conflicts of Interest: The authors declare no conflict of interest. The funders had no role in the design of the study; in the collection, analyses, or interpretation of data; in the writing of the manuscript, or in the decision to publish the results.

\section{References}

1. Interagency Coordination Group on Antimicrobial Resistance. No Time To Wait: Infections From Drug-Resistant Securing the Future; United Nations Foundation for the IACG: Geneva, Swizerland, 2019.

2. Kourtis, A.P.; Hatfield, K.; Baggs, J.; Mu, Y.; See, I.; Epson, E.; Nadle, J.; Kainer, M.A.; Dumyati, G.; Petit, S.; et al. Vital Signs: Epidemiology and Recent Trends in Methicillin-Resistant and in Methicillin-Susceptible Staphylococcus aureus Bloodstream Infections - United States. MMWR. Morb. Mortal. Wkly. Rep. 2019, 68, 214-219. [CrossRef] [PubMed]

3. Robert Koch-Institut. Infektionsepidemiologisches Jahrbuch meldepflichtiger Krankheiten für 2017; Krankenhaus-Hygiene + Infekt: Berlin, Germany, 2018.

4. Wos-Oxley, M.L.; Plumeier, I.; Von Eiff, C.; Taudien, S.; Platzer, M.; Vilchez-Vargas, R.; Becker, K.; Pieper, D.H. A poke into the diversity and associations within human anterior nare microbial communities. ISME J. 2010, 4, 839-851. [CrossRef] [PubMed]

5. Camelo-Castillo, A.; Henares, D.; Brotons, P.; Galiana, A.; Rodríguez, J.C.; Mira, A.; Muñoz-Almagro, C. Nasopharyngeal Microbiota in Children With Invasive Pneumococcal Disease: Identification of Bacteria With Potential Disease-Promoting and Protective Effects. Front. Microbiol. 2019, 10, 11. [CrossRef] [PubMed]

6. van Alen, S.; Ballhausen, B.; Peters, G.; Friedrich, A.W.; Mellmann, A.; Köck, R.; Becker, K. In the centre of an epidemic: Fifteen years of LA-MRSA CC398 at the University Hospital Münster. Vet. Microbiol. 2017, 200, 19-24. [CrossRef] [PubMed]

7. van Cleef, B.A.; Verkade, E.J.M.; Wulf, M.W.; Buiting, A.G.; Voss, A.; Huijsdens, X.W.; van Pelt, W.; Mulders, M.N.; Kluytmans, J.A. Prevalence of Livestock-Associated MRSA in Communities with High Pig-Densities in The Netherlands. PLoS ONE 2010, 5, e9385. [CrossRef] [PubMed]

8. Lewis, H.C.; Mølbak, K.; Reese, C.; Aarestrup, F.M.; Selchau, M.; Sørum, M.; Skov, R.L. Pigs as Source of Methicillin-Resistant Staphylococcus aureus CC398 Infections in Humans, Denmark. Emerg. Infect. Dis. 2008, 14, 1383-1389. [CrossRef] [PubMed]

9. Kaspar, U.; von Lützau, A.; Schlattmann, A.; Rösler, U.; Köck, R.; Becker, K. Zoonotic multidrug-resistant microorganisms among small companion animals in Germany. PLoS ONE 2018, 13, e0208364. [CrossRef]

10. Kaspar, U.; von Lützau, K.; Schlattmann, A.; Rösler, U.; Köck, R.; Becker, K. Zoonotic multidrug-resistant microorganisms among non-hospitalized horses from Germany. One Health 2019, 7, 100091. [CrossRef]

11. Cuny, C.; Friedrich, A.; Kozytska, S.; Layer, F.; Nübel, U.; Ohlsen, K.; Strommenger, B.; Walther, B.; Wieler, L.; Witte, W. Emergence of methicillin-resistant Staphylococcus aureus (MRSA) in different animal species. Int. J. Med. Microbiol. 2010, 300, 109-117. [CrossRef]

12. Becker, K.; Ballhausen, B.; Kahl, B.C.; Köck, R. The clinical impact of livestock-associated methicillin-resistant Staphylococcus aureus of the clonal complex 398 for humans. Vet. Microbiol. 2017, 200, 33-38. [CrossRef]

13. Köck, R.; Ballhausen, B.; Bischoff, M.; Cuny, C.; Eckmanns, T.; Fetsch, A.; Harmsen, D.; Goerge, T.; Oberheitmann, B.; Schwarz, S.; et al. The impact of zoonotic MRSA colonization and infection in Germany. Berl. Munch. Tierarztl. Wochenschr. 2014, 127, 384-398. [PubMed] 
14. Köck, R.; Schaumburg, F.; Mellmann, A.; Köksal, M.; Jurke, A.; Becker, K.; Friedrich, A.W. Livestock-Associated Methicillin-Resistant Staphylococcus aureus (MRSA) as Causes of Human Infection and Colonization in Germany. PLoS ONE 2013, 8, e55040. [CrossRef] [PubMed]

15. Schaumburg, F.; Köck, R.; Mellmann, A.; Richter, L.; Hasenberg, F.; Kriegeskorte, A.; Friedrich, A.W.; Gatermann, S.; Peters, G.; Von Eiff, C.; et al. Population dynamics among methicillin-resistant Staphylococcus aureus isolates in Germany during a 6-year period. J. Clin. Microbiol. 2012, 50, 3186-3192. [CrossRef] [PubMed]

16. Kaiser, A.M.; Haenen, A.J.P.; de Neeling, A.J.; Vandenbroucke-Grauls, C.M.J.E. Prevalence of Methicillin-Resistant Staphylococcus aureus and Risk Factors for Carriage in Dutch Hospitals. Infect. Control Hosp. Epidemiol. 2010, 31, 1188-1190. [CrossRef] [PubMed]

17. Sieber, R.N.; Skov, R.L.; Nielsen, J.; Schulz, J.; Price, L.B.; Aarestrup, F.M.; Larsen, A.R.; Stegger, M.; Larsen, J. Drivers and dynamics of methicillin-resistant livestock-associated Staphylococcus aureus CC398 in pigs and humans in Denmark. MBio 2018, 9, e02142-18. [CrossRef]

18. Schlattmann, A.; von Lützau, K.; Kaspar, U.; Becker, K. 'Rothia nasisuis' sp. nov., 'Dermabacter porcinasus' sp. nov., 'Propionibacterium westphaliense' sp. nov. and 'Tessaracoccus nasisuum' sp. nov., isolated from porcine nasal swabs in the Münster region, Germany. New Microbes New Infect. 2018, 26, 114-117. [CrossRef]

19. Idelevich, E.A.; Schüle, I.; Grünastel, B.; Wüllenweber, J.; Peters, G.; Becker, K. Rapid identification of microorganisms from positive blood cultures by MALDI-TOF mass spectrometry subsequent to very short-term incubation on solid medium. Clin. Microbiol. Infect. 2014, 20, 1001-1006. [CrossRef]

20. Stackebrandt, E.; Ebers, J. Taxonomic Parameters Revisited: Tarnished Gold Standards. Microbiol. Today 2006, 33, 152-155.

21. Cole, J.R.; Wang, Q.; Fish, J.A.; Chai, B.; McGarrell, D.M.; Sun, Y.; Brown, C.T.; Porras-Alfaro, A.; Kuske, C.R.; Tiedje, J.M. Ribosomal Database Project: Data and tools for high throughput rRNA analysis. Nucleic Acids Res. 2014, 42, D633-D642. [CrossRef]

22. European Committee on Antimicrobial Susceptibility Testing. Breakpoint Tables for Interpretation of MICs and Zone Diameters. Version 8.0. 2018. Available online: http//www.eucast.org (accessed on 1 January 2018).

23. Kriegeskorte, A.; Ballhausen, B.; Idelevich, E.A.; Köck, R.; Friedrich, A.W.; Karch, H.; Peters, G.; Becker, K. Human MRSA isolates with novel genetic homolog, Germany. Emerg. Infect. Dis. 2012, 18, 1016-1018. [CrossRef]

24. Becker, K.; van Alen, S.; Idelevich, E.A.; Schleimer, N.; Seggewiß, J.; Mellmann, A.; Kaspar, U.; Peters, G. Plasmid-Encoded Transferable mecB-Mediated Methicillin Resistance in Staphylococcus aureus. Emerg. Infect. Dis. 2018, 24, 242-248. [CrossRef] [PubMed]

25. Schwendener, S.; Cotting, K.; Perreten, V. Novel methicillin resistance gene mecD in clinical Macrococcus caseolyticus strains from bovine and canine sources. Sci. Rep. 2017, 7, 43797. [CrossRef] [PubMed]

26. Mellmann, A.; Weniger, T.; Berssenbrügge, C.; Keckevoet, U.; Friedrich, A.W.; Harmsen, D.; Grundmann, H. Characterization of Clonal Relatedness among the Natural Population of Staphylococcus aureus Strains by Using spa Sequence Typing and the BURP (Based upon Repeat Patterns) Algorithm. J. Clin. Microbiol. 2008, 46, 2805-2808. [CrossRef] [PubMed]

27. Quast, C.; Pruesse, E.; Yilmaz, P.; Gerken, J.; Schweer, T.; Yarza, P.; Peplies, J.; Glöckner, F.O. The SILVA ribosomal RNA gene database project: Improved data processing and web-based tools. Nucleic Acids Res. 2012, 41, D590-D596. [CrossRef] [PubMed]

28. Clarke, K.R. Non-parametric multivariate analyses of changes in community structure. Aust. J. Ecol. 1993, 18, 117-143. [CrossRef]

29. Clarke, K.R.; Gorley, R.N. PRIMER v6: User Manual/Tutorial; PRIMER-E: Plymouth, UK, 2006; p. 190.

30. Lowe, B.A.; Marsh, T.L.; Isaacs-Cosgrove, N.; Kirkwood, R.N.; Kiupel, M.; Mulks, M.H. Defining the "core microbiome" of the microbial communities in the tonsils of healthy pigs. BMC Microbiol. 2012, 12, 20. [CrossRef]

31. Weese, J.S.; Slifierz, M.; Jalali, M.; Friendship, R. Evaluation of the nasal microbiota in slaughter-age pigs and the impact on nasal methicillin-resistant Staphylococcus aureus (MRSA) carriage. BMC Vet. Res. 2014, 10, 69. [CrossRef]

32. Strube, M.L.; Hansen, J.E.; Rasmussen, S.; Pedersen, K. A detailed investigation of the porcine skin and nose microbiome using universal and Staphylococcus specific primers. Sci. Rep. 2018, 8, 12751. [CrossRef] 
33. Bhargava, K.; Zhang, Y. Characterization of methicillin-resistant coagulase-negative staphylococci (MRCoNS) in retail meat. Food Microbiol. 2014, 42, 56-60. [CrossRef]

34. Zong, Z.; Peng, C.; Lü, X. Diversity of SCCmec elements in methicillin-resistant coagulase-negative staphylococci clinical isolates. PLoS ONE 2011, 6, e20191. [CrossRef]

35. Nemeghaire, S.; Vanderhaeghen, W.; Argudin, M.A.; Haesebrouck, F.; Butaye, P. Characterization of methicillin-resistant Staphylococcus sciuri isolates from industrially raised pigs, cattle and broiler chickens. J. Antimicrob. Chemother. 2014, 69, 2928-2934. [CrossRef] [PubMed]

36. Kaspar, U.; Kriegeskorte, A.; Schubert, T.; Peters, G.; Rudack, C.; Pieper, D.H.; Wos-Oxley, M.; Becker, K. The culturome of the human nose habitats reveals individual bacterial fingerprint patterns. Environ. Microbiol. 2016, 18, 2130-2142. [CrossRef] [PubMed]

37. Sahibzada, S.; Hernández-Jover, M.; Jordan, D.; Thomson, P.C.; Heller, J. Emergence of highly prevalent CA-MRSA ST93 as an occupational risk in people working on a pig farm in Australia. PLoS ONE 2018, 13, e0195510. [CrossRef] [PubMed]

38. Parada, J.L.; Caron, C.R.; Medeiros, A.B.P.; Soccol, C.R. Bacteriocins from lactic acid bacteria: Purification, properties and use as biopreservatives. Braz. Arch. Biol. Technol. 2007, 50, 521-542. [CrossRef]

39. Zipperer, A.; Konnerth, M.C.; Laux, C.; Berscheid, A.; Janek, D.; Weidenmaier, C.; Burian, M.; Schilling, N.A.; Slavetinsky, C.; Marschal, M.; et al. Human commensals producing a novel antibiotic impair pathogen colonization. Nature 2016, 535, 511-516. [CrossRef]

40. Schmithausen, R.M.; Schulze-Geisthoevel, S.V.; Stemmer, F.; El-Jade, M.; Reif, M.; Hack, S.; Meilaender, A.; Montabauer, G.; Fimmers, R.; Parcina, M.; et al. Analysis of Transmission of MRSA and ESBL-E among Pigs and Farm Personnel. PLoS ONE 2015, 10, e0138173. [CrossRef]

41. Fetsch, A.; Roesler, U.; Kraushaar, B.; Friese, A. Co-colonization and clonal diversity of methicillin-sensitive and methicillin-resistant Staphylococcus aureus in sows. Vet. Microbiol. 2016, 185, 7-14. [CrossRef]

42. Schoenfelder, S.M.K.; Dong, Y.; Feßler, A.T.; Schwarz, S.; Schoen, C.; Köck, R.; Ziebuhr, W. Antibiotic resistance profiles of coagulase-negative staphylococci in livestock environments. Vet. Microbiol. 2017, 200, 79-87. [CrossRef]

43. Dahms, C.; Hübner, N.-O.; Kossow, A.; Mellmann, A.; Dittmann, K.; Kramer, A. Occurrence of ESBL-Producing Escherichia coli in Livestock and Farm Workers in Mecklenburg-Western Pomerania, Germany. PLoS ONE 2015, 10, e0143326. [CrossRef]

44. Hooper, D.C.; Jacoby, G.A. Mechanisms of drug resistance: Quinolone resistance. Ann. N. Y. Acad. Sci. 2015, 1354, 12-31. [CrossRef]

45. Federal Office of Consumer Protection and Food Safety (BVL). Erneut weniger Antibiotika in der Tiermedizin abgegeben. Available online: https://www.bvl.bund.de/SharedDocs/Pressemitteilungen/05_tierarzneimittel/ 2019/2019_07_25_PI_Antibiotikaabgabe.html (accessed on 10 December 2019).

46. Lloyd, D.H.; Page, S.W. Antimicrobial Stewardship in Veterinary Medicine. Microbiol. Spectr. $2018,6$. [CrossRef] [PubMed]

(C) 2020 by the authors. Licensee MDPI, Basel, Switzerland. This article is an open access article distributed under the terms and conditions of the Creative Commons Attribution (CC BY) license (http://creativecommons.org/licenses/by/4.0/). 